\section{Tolerance of Four Festuca Species to Ethofumesate and Prodiamine}

\author{
Peter H. Dernoeden ${ }^{1}$ \\ Department of Natural Resource Sciences and Landscape Architecture, \\ University of Maryland, College Park, MD 20742-5821
}

Additional index words. herbicides, turfgrasses, phytotoxicity, dithiopyr

\begin{abstract}
Festuca species are being seeded into golf course roughs and natural or out-ofbound areas as alternative turfgrasses to replace perennial ryegrass (Lolium perenne $\mathbf{L}$.) in the mid-Atlantic region. The tolerance of fine-leaf fescues to herbicides targeted for annual bluegrass (Poa annua L.) control, such as ethofumesate and prodiamine, is unknown. The objectives of this field study, therefore, were to assess the tolerance of 'Rebel II' tall fescue (Festuca arundinacea Schreb.), and the fine-leaf fescue species 'Reliant' hard fescue (Festuca longifolia Thuill.), 'Jamestown II' Chewings fescue (Festuca rubra L. ssp. commutata Gaud.), and 'MX 86' blue sheep fescue (Festuca glauca L.) to various rates, combinations, and times of application of ethofumesate and prodiamine. 'Rebel II' was most tolerant of ethofumesate; however, sequential rates $\geq 0.84+0.84 \mathrm{~kg} \cdot \mathrm{ha}^{-1}$ reduced quality for 1 or more weeks and $2.24+2.24 \mathrm{~kg} \cdot \mathrm{ha}^{-1}$ caused unacceptable injury. Single applications of ethofumesate at rates of $0.56,0.84$, and $1.12 \mathrm{~kg} \cdot \mathrm{ha}^{-1}$, and sequential treatments of $0.56+0.56$ and $0.84+0.84 \mathrm{~kg}^{\prime} \mathrm{ha}^{-1}$ reduced 'Reliant' quality temporarily. Sequential treatments of high rates (i.e., $1.12+1.12$ and $2.24+2.24 \mathrm{~kg}^{\circ} \mathrm{ha}^{-1}$ ), however, significantly reduced 'Reliant' cover. 'Jamestown II' was very sensitive to ethofumesate, but recovered from single applications of $0.56,0.84$, and $1.12 \mathrm{~kg} \cdot \mathrm{ha}^{-1}$; sequential applications $\left(\geq 0.84+0.84 \mathrm{~kg} \cdot \mathrm{ha}^{-1}\right)$ caused unacceptable injury, and rates $\geq 1.12+1.12 \mathrm{~kg} \cdot \mathrm{ha}^{-1}$ caused significant loss of cover. The cultivar MX 86 tolerated single applications of 0.56 to $2.24 \mathrm{~kg} \cdot \mathrm{ha}^{-1}$ of ethofumesate, but sequential treatments generally reduced quality to unacceptable levels. In one study, 'Jamestown II' and 'MX 86' were more severely injured when ethofumesate $\left(1.12\right.$ or $\left.2.24 \mathrm{~kg} \cdot \mathrm{ha}^{-1}\right)$ was applied in October rather than in November. The fescues generally best tolerated a single, November application of ethofumesate at $\leq 1.12 \mathrm{~kg} \cdot \mathrm{ha}^{-1}$. Prodiamine $\left(0.73 \mathrm{~kg} \cdot \mathrm{ha}^{-1}\right)$ caused only short-term reductions in quality of 'Jamestown II', but was generally noninjurious to the other fescues. Ethofumesate tank-

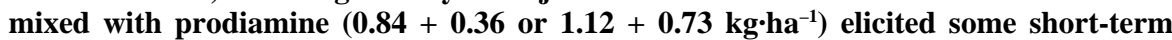
reduction in quality, but the level of injury was generally acceptable and injured fescues had recovered by spring. Chemical names used: $[ \pm] 2$-ethoxy-2,3-dihydro-3,3-dimethyl-5benzofuranyl methanesulfonate (ethofumesate); $N^{3}, N^{3}$-di-n-propyl-2,4-dinitro-6(trifluoromethyl)-m-phenylenediamine (prodiamine); $S, S$-dimethyl 2-(difluoromethyl)4-(2-methylpropyl)-6-(trifluoromethyl)-3,5-pyridine-dicarbothioate (dithiopyr).
\end{abstract}

Golf course superintendents in the midAtlantic region are searching for turfgrass species that will perform well with reduced management inputs in roughs and infrequently mowed natural areas. Research conducted in Maryland indicated that 'Reliant' hard fescue, 'Bighorn' blue sheep fescue, and 'Rebel II' tall fescue provided good quality under low maintenance (Dernoeden et al., 1998b). Perennial ryegrass is the dominant species grown in roughs in the region, but it was decimated by gray leaf spot [Pyricularia grisea (Cooke) Sacc.] epidemics in 1995 and 1998, and ice damage in the 1993-94 winter. Hard and blue sheep fescues are better suited to natural or out-of-play areas where there is little traffic or need for mowing during the summer (Dernoeden, 1998b). Tall fescue has better

Received for publication 23 Sept. 1999. Accepted for publication 9 Feb. 2000. This manuscript is a contribution from the Maryland Agriculture Experiment Station. The cost of publishing this paper was defrayed in part by the payment of page charges. Under postal regulations, this paper therefore must be hereby marked advertisement solely to indicate this fact.

${ }^{1}$ E-mail address: pd9@umail.umd.edu summer mowing and wear tolerance than the aforementioned fescues, and would be suitable for rough areas mowed above $6.0 \mathrm{~cm}$.

Interest in using fescue species for golf course roughs and other low-maintenance situations is increasing, but little is known regarding their tolerance to commonly used herbicides. Annual bluegrass is a major golf course weed problem, and huge reservoirs of seed are produced in roughs. Common herbicides used for annual bluegrass control in the region include ethofumesate (ETHO) and prodiamine, which are used postemergence and preemergence, respectively. Both chemicals generally are applied between late summer and early winter (Dernoeden, 1998a; Dernoeden and Turner, 1988); therefore, grasses grown in roughs and natural areas on golf courses should be tolerant of these herbicides. Among fescue species, ETHO is labeled for use only on tall fescue. The focus of this study was to assess the tolerance of four fescue species to ETHO and prodiamine alone and to mixtures of both chemicals, as the combination of herbicides may provide early postemergence and subsequent preemergence control of annual bluegrass.
Monostands of 'Jamestown II' Chewings fescue, 'Rebel II' tall fescue, 'Reliant' hard fescue, and 'MX 86' blue sheep fescue were established in Sept. 1995. The soil was a Chillum silt loam (fine silty, mixed mesic Typic Hapludult) with a pH of 5.5 and $21 \mathrm{mg}$ organic matter per gram of soil. The monostands were fertilized in spring and fall with $50 \mathrm{~kg} \cdot \mathrm{ha}^{-1} \mathrm{~N}$ from urea. Initial soil $\mathrm{P}$ and $\mathrm{K}$ levels were 268 and $431 \mathrm{~kg} \cdot \mathrm{ha}^{-1}$, respectively. All stands were mowed to a height of $6.5 \pm 0.5 \mathrm{~cm}$, but unless otherwise stated they were not irrigated during the study period. No other pesticides were applied to the research sites during the study.

In 1996, herbicides were applied 1 Oct., with four sequential ETHO treatments applied on 22 Oct. The sites used in 1996 were rerandomized and treated in 1997. Plots that were severely injured from the 1996 study were eliminated, but there were enough healthy, high-density plots present within each site to accommodate all 1997 treatments. In 1997, different rates of the herbicides were assessed alone or in combination. Two application dates (October vs. November) were compared in 1997 to determine if phytotoxicity were influenced by application timing. All monostands were irrigated within $48 \mathrm{~h}$ of herbicide treatment. Plots were $1.5 \times 1.5 \mathrm{~m}$, and were arranged in a randomized complete block with three replications. Turf quality was assessed visually using a 0 to 10 scale where $0=$ entire plot area brown or dead, 7.0 = minimum acceptable quality for golf course roughs, and 10 $=$ optimum color and density. Percentage of plot area brown, dead, or bareground was rated on a visual $0 \%$ to $100 \%$ linear scale where $0=$ entire plot area green and healthy, and $100=$ entire plot area brown, dead, or bareground. Data were subjected to analysis of variance and significantly different means were separated at $P \leq 0.05$ by the least significant difference $t$ test.

\section{Results}

\section{Study I (1996-97)}

Data are shown only when treatment effects were significant. Dithiopyr was evaluated only in Study I. Dithiopyr and prodiamine applied alone caused no discoloration or loss of density. Ethofumesate data are discussed below for each cultivar. Most of the ETHOinduced injury took the form of an initial bluegreen or pale-green discoloration, followed in some cases by a browning of foliar tissues. In more extreme cases, plants were killed and this injury is reported as percentage of plot area bareground.

'Rebel II' tall fescue. Ethofumesate applied once did not reduce tall fescue quality significantly on any date (Table 1). Conversely, all sequential treatments reduced quality on at least one rating date. Rates $\geq 0.84+0.84$ $\mathrm{kg} \cdot \mathrm{ha}^{-1}$ reduced quality from 28 Oct. to $2 \mathrm{Dec}$. 1996, particularly in plots treated twice with $2.24 \mathrm{~kg} \cdot \mathrm{ha}^{-1}$. By $20 \mathrm{Feb} .1997$, reduced quality 
was observed only at the high sequential rate. While none of the treatments resulted in a loss of turf density (data not shown), the high sequential rate caused commercially unacceptable levels of discoloration. The ETHO + prodiamine tank-mix treatment did not injure 'Rebel II'.

'Reliant' hard fescue. 'Reliant' tolerated a single application of ETHO regardless of rate, but was injured by sequential treatments (Table 1). Most initial injury ( 28 Oct. and 4 Nov.) was evident with the higher two rates (1.12 and $\left.2.24 \mathrm{~kg} \cdot \mathrm{ha}^{-1}\right)$. The lower, sequential rate treatments, however, eventually caused discoloration and reduced quality from 12 Nov. 1996 to 20 Feb. 1997. Data collected 20 Feb. showed that all sequential ETHO treatments had reduced turf quality, particularly with $2.24+$ $2.24 \mathrm{~kg} \cdot \mathrm{ha}^{-1}$. All sequentially treated plots had unacceptable quality (i.e., $\leq 7.0$ ) on the final rating date, and therefore these treatments were judged to be too phytotoxic for hard fescue. The ETHO + prodiamine tank-mix did not adversely affect 'Reliant' quality.

'Jamestown II' Chewings fescue. Only the single, $0.56 \mathrm{~kg} \cdot \mathrm{ha}^{-1}$ rate of ETHO did not injure or reduce the quality of 'Jamestown II' (Table 2). A single application of high rates of ETHO generally reduced quality within a 2week period from 14 to 28 Oct. Sequentially treated plots showed some loss in quality, but quality was unacceptable on most rating dates at rates $\geq 0.84+0.84 \mathrm{~kg} \cdot \mathrm{ha}^{-1}$ from 28 Oct. 1996 to 20 Feb. 1997 (data for some dates not shown). On 6 May 1997, all plots had recovered except those sprayed with the two highest sequential rates. Plots treated with $1.12+1.12$ $\mathrm{kg} \cdot \mathrm{ha}^{-1}$ had $8 \%$ bareground, whereas the highest sequential rate had caused a $70 \%$ loss of 'Jamestown II' cover (data not shown). 'Jamestown II' plots treated with $0.56+0.56$ $\mathrm{kg} \cdot \mathrm{ha}^{-1}$ of ETHO had marginally unacceptable quality, but higher rates caused an unacceptable loss of quality and/or cover. The ETHO + prodiamine tank-mix was relatively safe, but had reduced quality when plots were rated on 14 and 28 Oct.

'MX 86 ' blue sheep fescue. The $2.24 \mathrm{~kg} \cdot \mathrm{ha}^{-1}$ ETHO rate was the only single application treatment that had reduced quality as of 28 Oct. (Table 2). All sequential treatments reduced quality from 28 Oct. 1996 to 20 Feb. 1997 , with the $2.24+2.24 \mathrm{~kg} \cdot \mathrm{ha}^{-1}$ treatment causing significant turf loss $(7 \%$ bareground, data not shown). The ETHO + prodiamine tank-mix was noninjurious.

\section{Study II (1997-98)}

The injury associated with the sequential treatments in Study I was probably the result of higher total amounts of herbicide being applied. However, fescue sensitivity to ETHO also may have been influenced by the time of application. Therefore, in Study II, the high ETHO rates (1.12 and $2.24 \mathrm{~kg} \cdot \mathrm{ha}^{-1}$ ) were applied once in either October or November, whereas the lower rates were reevaluated only as sequential treatments.

'Rebel II' tall fescue. Significant injury was evident from 23 Oct. through $5 \mathrm{Dec}$. in plots treated with either 1.12 or $2.24 \mathrm{~kg} \cdot \mathrm{ha}^{-1}$ of ETHO (Table 3, some data not shown), but quality was equivalent to that of nontreated plots by 14 Jan. 1998. Among the other treatments, only ETHO + prodiamine applied in November had reduced quality on 19 Nov. and 5 Dec., and the tank-mix applied in October at $1.12+0.73 \mathrm{~kg} \cdot \mathrm{ha}^{-1}$ had reduced quality on 14 Jan. None of the treatments reduced quality to unacceptable levels, and quality in all herbicide-treated plots was equivalent to that of nontreated turf by 6 Feb. (data not shown).

'Reliant' hard fescue. Ethofumesate applied alone on 8 Oct. at 1.12 and $2.24 \mathrm{~kg} \cdot \mathrm{ha}^{-1}$ reduced quality by 23 Oct. (data not shown). Plots treated with $2.24 \mathrm{~kg} \cdot \mathrm{ha}^{-1}$ alone generally were more severely damaged than those sprayed with $1.12 \mathrm{~kg} \cdot \mathrm{ha}^{-1}$ from 19 Nov. to 6 Mar. (Table 3). Turf in plots treated with 1.12 $\mathrm{kg} \cdot \mathrm{ha}^{-1}$ had recovered by 14 Jan. 1998 . The sequential ETHO $\left(0.84+0.84 \mathrm{~kg} \cdot \mathrm{ha}^{-1}\right)$ and $\mathrm{ETHO}+$ prodiamine treatments, except $0.84+$

Table 1. 'Rebel II' tall fescue and 'Reliant' hard fescue quality ${ }^{\mathrm{z}}$ as influenced by ethofumesate and prodiamine application, $1996-97$.

\begin{tabular}{|c|c|c|c|c|c|c|c|c|c|c|c|c|}
\hline \multirow[b]{3}{*}{ Herbicide } & \multirow{2}{*}{\multicolumn{2}{|c|}{$\begin{array}{c}\text { Rate } \\
\left(\mathrm{kg} \cdot \mathrm{ha}^{-1}\right)\end{array}$}} & \multicolumn{5}{|c|}{ Rebel II } & \multicolumn{5}{|c|}{ Reliant } \\
\hline & & & \multicolumn{4}{|c|}{1996} & \multirow{2}{*}{$\frac{1997}{20 \mathrm{Feb} .}$} & \multicolumn{4}{|c|}{1996} & \multirow{2}{*}{$\frac{1997}{20 \mathrm{Feb}}$} \\
\hline & 1 Oct. & 22 Oct. & 28 Oct. & 4 Nov. & 12 Nov. & 2 Dec. & & 28 Oct. & 4 Nov. & 12 Nov. & 2 Dec. & \\
\hline \multirow[t]{8}{*}{ Ethofumesate } & 0.56 & --- & $8.7 \mathrm{a}^{\mathrm{y}}$ & $8.6 \mathrm{a}$ & $8.7 \mathrm{a}$ & $8.2 \mathrm{ab}$ & $6.5 \mathrm{ab}$ & $8.6 \mathrm{ab}$ & $8.8 \mathrm{ab}$ & $8.6 \mathrm{a}$ & $8.8 \mathrm{a}$ & $7.6 \mathrm{a}$ \\
\hline & 0.84 & --- & $8.6 \mathrm{a}$ & $8.4 \mathrm{a}$ & $8.5 \mathrm{ab}$ & $8.2 \mathrm{ab}$ & $6.5 \mathrm{ab}$ & $8.5 \mathrm{a}-\mathrm{c}$ & $8.8 \mathrm{ab}$ & $8.7 \mathrm{a}$ & $8.8 \mathrm{a}$ & $7.4 \mathrm{a}$ \\
\hline & 1.12 & --- & $8.4 \mathrm{ab}$ & $8.6 \mathrm{a}$ & $8.4 \mathrm{ab}$ & $8.2 \mathrm{ab}$ & $6.6 \mathrm{a}$ & $8.6 \mathrm{ab}$ & $8.8 \mathrm{ab}$ & $8.6 \mathrm{a}$ & $8.6 \mathrm{a}$ & $7.5 \mathrm{a}$ \\
\hline & 2.24 & --- & $8.4 \mathrm{ab}$ & $8.4 \mathrm{a}$ & $8.2 \mathrm{~b}$ & $8.2 \mathrm{ab}$ & $6.5 \mathrm{ab}$ & $8.7 \mathrm{ab}$ & $8.9 \mathrm{a}$ & $8.6 \mathrm{a}$ & $8.7 \mathrm{a}$ & $7.6 \mathrm{a}$ \\
\hline & 0.56 & 0.56 & $8.0 \mathrm{~b}$ & $8.4 \mathrm{a}$ & $8.4 \mathrm{ab}$ & $8.2 \mathrm{ab}$ & $6.6 \mathrm{a}$ & $8.4 \mathrm{bc}$ & $8.4 \mathrm{a}-\mathrm{c}$ & $7.8 \mathrm{~b}$ & $7.9 \mathrm{~b}$ & $6.9 \mathrm{~b}$ \\
\hline & 0.84 & 0.84 & $7.2 \mathrm{c}$ & $7.7 \mathrm{~b}$ & $7.8 \mathrm{c}$ & $7.9 \mathrm{~b}$ & $6.4 \mathrm{~b}$ & $8.2 \mathrm{ab}$ & $8.1 \mathrm{~cd}$ & $6.4 \mathrm{c}$ & $6.3 \mathrm{c}$ & $6.4 \mathrm{c}$ \\
\hline & 1.12 & 1.12 & $7.1 \mathrm{c}$ & $7.1 \mathrm{c}$ & $7.6 \mathrm{c}$ & $7.9 \mathrm{~b}$ & $6.4 \mathrm{~b}$ & $8.2 \mathrm{c}$ & $7.8 \mathrm{~d}$ & $6.1 \mathrm{c}$ & $6.1 \mathrm{c}$ & $6.0 \mathrm{c}$ \\
\hline & 2.24 & 2.24 & $6.1 \mathrm{~d}$ & $5.6 \mathrm{~d}$ & $6.0 \mathrm{~d}$ & $7.4 \mathrm{c}$ & $6.0 \mathrm{c}$ & $7.7 \mathrm{~d}$ & $6.1 \mathrm{e}$ & $2.8 \mathrm{~d}$ & $2.8 \mathrm{~d}$ & $4.0 \mathrm{~d}$ \\
\hline Prodiamine & 0.73 & --- & $8.5 \mathrm{a}$ & $8.5 \mathrm{a}$ & $8.5 \mathrm{ab}$ & $8.2 \mathrm{ab}$ & $6.4 \mathrm{~b}$ & $8.6 \mathrm{ab}$ & $8.6 \mathrm{ab}$ & $8.6 \mathrm{a}$ & $8.6 \mathrm{a}$ & $7.5 \mathrm{a}$ \\
\hline Dithiopyr & 0.56 & --- & $8.6 \mathrm{a}$ & $8.5 \mathrm{a}$ & $8.4 \mathrm{ab}$ & $8.4 \mathrm{a}$ & $6.5 \mathrm{ab}$ & $8.4 \mathrm{bc}$ & $8.8 \mathrm{ab}$ & $8.6 \mathrm{a}$ & $8.7 \mathrm{a}$ & $7.6 \mathrm{a}$ \\
\hline Ethofumesate + & $0.84+$ & & & & & & & & & & & \\
\hline prodiamine & 0.36 & --- & $8.6 \mathrm{a}$ & $8.5 \mathrm{a}$ & $8.4 \mathrm{ab}$ & $8.1 \mathrm{ab}$ & $6.5 \mathrm{ab}$ & $8.8 \mathrm{a}$ & $8.8 \mathrm{ab}$ & $8.4 \mathrm{a}$ & $8.5 \mathrm{a}$ & $7.7 \mathrm{a}$ \\
\hline Not treated & --- & --- & $8.6 \mathrm{a}$ & $8.4 \mathrm{a}$ & $8.5 \mathrm{ab}$ & $8.2 \mathrm{ab}$ & $6.5 \mathrm{ab}$ & $8.6 \mathrm{ab}$ & $8.7 \mathrm{ab}$ & $8.6 \mathrm{a}$ & $8.8 \mathrm{a}$ & $7.6 \mathrm{a}$ \\
\hline
\end{tabular}

${ }^{2}$ Turf quality was assessed visually using a 0 to 10 scale where $0=$ entire plot area brown or dead, and $10=$ optimum color and density.

${ }^{\mathrm{y}}$ Mean separation within columns by LSD, $P \leq 0.05$.

Table 2. 'Jamestown II' Chewings fescue and 'MX 86' blue sheep fescue quality ${ }^{\mathrm{z}}$ as influenced by ethofumesate and prodiamine application, 1996 and 1997.

\begin{tabular}{|c|c|c|c|c|c|c|c|c|c|c|c|}
\hline \multirow[b]{3}{*}{ Treatment } & \multirow{2}{*}{\multicolumn{2}{|c|}{$\begin{array}{c}\text { Rate } \\
\left(\mathrm{kg} \cdot \mathrm{ha}^{-1}\right)\end{array}$}} & \multicolumn{5}{|c|}{ Jamestown II } & \multicolumn{4}{|c|}{ MX 86} \\
\hline & & & \multicolumn{2}{|c|}{1996} & \multicolumn{3}{|c|}{1997} & \multicolumn{2}{|c|}{1996} & \multicolumn{2}{|c|}{1997} \\
\hline & 1 Oct. & 22 Oct. & 14 Oct. & 28 Oct. & 4 Nov. & 2 Dec. & 20 Feb. & 28 Oct. & 4 Nov. & 2 Dec. & 20 Feb. \\
\hline \multirow[t]{8}{*}{ Ethofumesate } & 0.56 & --- & $8.8 \mathrm{a}^{\mathrm{y}}$ & $8.6 \mathrm{ab}$ & $8.8 \mathrm{a}$ & $8.6 \mathrm{a}$ & $7.5 \mathrm{a}$ & $8.0 \mathrm{a}-\mathrm{c}$ & $8.6 \mathrm{a}$ & $8.6 \mathrm{a}$ & $7.0 \mathrm{a}$ \\
\hline & 0.84 & --- & $8.4 \mathrm{c}-\mathrm{e}$ & $8.3 \mathrm{~b}-\mathrm{d}$ & $8.3 \mathrm{a}-\mathrm{c}$ & $8.5 \mathrm{ab}$ & $7.5 \mathrm{a}$ & $8.2 \mathrm{ab}$ & $8.6 \mathrm{a}$ & $8.4 \mathrm{a}$ & $6.9 \mathrm{a}$ \\
\hline & 1.12 & --- & $8.2 \mathrm{de}$ & $8.1 \mathrm{c}-\mathrm{e}$ & $8.5 \mathrm{ab}$ & $8.5 \mathrm{ab}$ & $7.5 \mathrm{a}$ & $8.0 \mathrm{a}-\mathrm{c}$ & $8.6 \mathrm{a}$ & $8.6 \mathrm{a}$ & $7.0 \mathrm{a}$ \\
\hline & 2.24 & --- & $8.2 \mathrm{de}$ & $7.8 \mathrm{e}$ & $8.1 \mathrm{bc}$ & $8.0 \mathrm{~b}$ & $7.0 \mathrm{ab}$ & $7.8 \mathrm{~b}-\mathrm{d}$ & $8.5 \mathrm{a}$ & $8.6 \mathrm{a}$ & $7.0 \mathrm{a}$ \\
\hline & 0.56 & 0.56 & $8.6 \mathrm{a}-\mathrm{d}$ & $8.0 \mathrm{de}$ & $7.7 \mathrm{c}$ & $8.0 \mathrm{~b}$ & $6.6 \mathrm{~b}$ & $7.5 \mathrm{de}$ & $7.8 \mathrm{~b}$ & $7.4 \mathrm{~b}$ & $6.5 \mathrm{~b}$ \\
\hline & 0.84 & 0.84 & $8.5 \mathrm{a}-\mathrm{e}$ & $7.0 \mathrm{f}$ & $6.4 \mathrm{~d}$ & $5.2 \mathrm{c}$ & $5.1 \mathrm{c}$ & $7.1 \mathrm{fg}$ & $6.5 \mathrm{c}$ & $5.6 \mathrm{c}$ & $5.5 \mathrm{c}$ \\
\hline & 1.12 & 1.12 & $8.2 \mathrm{de}$ & $6.4 \mathrm{~g}$ & $5.3 \mathrm{e}$ & $2.5 \mathrm{~d}$ & $2.8 \mathrm{~d}$ & $6.9 \mathrm{~g}$ & $6.2 \mathrm{c}$ & $5.2 \mathrm{c}$ & $5.3 \mathrm{c}$ \\
\hline & 2.24 & 2.24 & $8.1 \mathrm{e}$ & $5.1 \mathrm{~h}$ & $2.8 \mathrm{f}$ & $0.0 \mathrm{e}$ & $0.0 \mathrm{e}$ & $6.4 \mathrm{~h}$ & $5.2 \mathrm{~d}$ & $1.8 \mathrm{~d}$ & $2.2 \mathrm{~d}$ \\
\hline Prodiamine & 0.73 & --- & $8.8 \mathrm{a}-\mathrm{c}$ & $8.5 \mathrm{a}-\mathrm{c}$ & $8.6 \mathrm{ab}$ & $8.2 \mathrm{ab}$ & $7.5 \mathrm{a}$ & $8.0 \mathrm{a}-\mathrm{c}$ & $8.7 \mathrm{a}$ & $8.6 \mathrm{a}$ & $7.0 \mathrm{a}$ \\
\hline Dithiopyr & 0.56 & --- & $8.4 \mathrm{~b}-\mathrm{e}$ & $8.2 \mathrm{c}-\mathrm{e}$ & $8.1 \mathrm{bc}$ & $8.4 \mathrm{ab}$ & $7.4 \mathrm{a}$ & $8.0 \mathrm{a}-\mathrm{c}$ & $8.6 \mathrm{a}$ & $8.4 \mathrm{a}$ & $6.9 \mathrm{a}$ \\
\hline $\begin{array}{c}\text { Ethofumesate }+ \\
\text { prodiamine }\end{array}$ & $\begin{array}{l}0.84+ \\
0.36\end{array}$ & --- & $8.2 \mathrm{de}$ & $8.0 \mathrm{de}$ & $8.3 \mathrm{a}-\mathrm{c}$ & $8.4 \mathrm{ab}$ & $7.3 \mathrm{a}$ & $8.1 \mathrm{ab}$ & $8.6 \mathrm{a}$ & $8.4 \mathrm{a}$ & $7.0 \mathrm{a}$ \\
\hline Not treated & --- & --- & $8.8 \mathrm{ab}$ & $8.6 \mathrm{ab}$ & $8.6 \mathrm{ab}$ & $8.5 \mathrm{ab}$ & $7.5 \mathrm{a}$ & $8.3 \mathrm{a}$ & $8.6 \mathrm{a}$ & $8.6 \mathrm{a}$ & $7.0 \mathrm{a}$ \\
\hline
\end{tabular}

${ }^{2}$ Turf quality was assessed visually using a 0 to 10 scale where $0=$ entire plot area brown or dead, and $10=$ optimum color and density.

yean separation within columns by LSD, $P \leq 0.05$. 
$0.36 \mathrm{~kg} \cdot \mathrm{ha}^{-1}$ applied in October, generally reduced quality from 19 Nov. 1997 to 6 Mar. 1998. Except for the November application evaluated on 6 Mar., plots treated with prodiamine alone exhibited no reduction in quality. While all herbicide-treated plots had similar levels of quality on $2 \mathrm{Apr}$. and 4 May 1998, plots treated with $2.24 \mathrm{~kg} \cdot \mathrm{ha}^{-1}$ ETHO in Oct. 1997 did not fully recover until 4 May 1998 (data not shown). None of the treatments caused reduced density significantly (data not shown).

'Jamestown II' Chewings fescue. Reduced turf quality was evident on 23 Oct. 1997 through 4 May 1998 in selected ETHO-treated plots, particularly at $2.24 \mathrm{~kg} \cdot \mathrm{ha}^{-1}$ (Table 4 , some data not shown). The 1.12 and $2.24 \mathrm{~kg} \cdot \mathrm{ha}^{-1}$ rates applied in October elicited more injury than the November application from 6 Feb. to 4 May. On the final rating date (4 May), only plots treated with $2.24 \mathrm{~kg} \cdot \mathrm{ha}^{-1}$ in October had quality ratings inferior to that of the nontreated control. Application of prodiamine in October did not reduce quality; however, application in
November reduced quality from 14 Jan. 1997 to 2 Apr. 1998, but ratings remained in the acceptable range (i.e., >7.0). Only the 2.24 $\mathrm{kg} \cdot \mathrm{ha}^{-1}$ rate of ETHO applied in October caused a significant loss of turf (4\% bareground) when plots were rated on 4 May 1998 (data not shown).

'MX 86' blue sheep fescue. The 1.12 and $2.24 \mathrm{~kg} \cdot \mathrm{ha}^{-1}$ rates of ETHO applied alone in October reduced quality on 23 Oct. (data not shown), and nearly all rates reduced quality from 19 Nov. to 6 Feb. (Table 4). Poorest quality was most often associated with the $2.24 \mathrm{~kg} \cdot \mathrm{ha}^{-1}$ treatment during this period. Except for the $1.12 \mathrm{~kg} \cdot \mathrm{ha}^{-1}$ (October timing) and the $2.24 \mathrm{~kg} \cdot \mathrm{ha}^{-1}$ rates (both timings), most treated plots had recovered by 6 Mar. At the final rating, only 1.12 and $2.24 \mathrm{~kg} \cdot \mathrm{ha}^{-1}$ applied in October had reduced quality ratings. Turf treated with $2.24 \mathrm{~kg} \cdot \mathrm{ha}^{-1}$ in October had the highest bareground ratings ( $12 \%$ ) on $6 \mathrm{Feb}$; by 4 May, significantly higher ratings were noted in plots treated in October with 1.12 (3\% bareground) and 2.24 (4\% bareground) $\mathrm{kg} \cdot \mathrm{ha}^{-1}$ (data not shown). Ethofumesate + prodiamine (all treatments) reduced quality on 5 Dec., but quality had generally recovered to control levels by 6 Feb. Prodiamine applied alone did not reduce quality or density on any date.

\section{Discussion}

Most ETHO treatments at rates $\geq 1.12$ $\mathrm{kg} \cdot \mathrm{ha}^{-1}$ reduced turf quality in all species on one or more rating dates. Prodiamine alone generally was safe, although it did cause some reduction in quality on a few rating dates in 'Jamestown II' and 'Reliant'. Ethofumesate + prodiamine and sequential treatment with ETHO reduced quality to levels similar to those obtained with ETHO applied alone at 1.12 $\mathrm{kg} \cdot \mathrm{ha}^{-1}$, and were generally safer than ETHO applied alone at $2.24 \mathrm{~kg} \cdot \mathrm{ha}^{-1}$. Ethofumesate + prodiamine reduced 'Reliant' quality to unacceptable levels in Study II on one rating date (6 Mar.), but loss of cover was nonsignificant for any of the fescues in any year.

'Rebel II' tolerated ETHO better than did

Table 3. 'Rebel II' tall fescue and 'Reliant' hard fescue quality² as influenced by ethofumesate and prodiamine application, $1997-98$.

\begin{tabular}{|c|c|c|c|c|c|c|c|c|c|c|}
\hline \multirow[b]{3}{*}{ Treatment } & \multirow{3}{*}{$\begin{array}{l}\text { Rate } \\
\left(\mathrm{kg} \cdot \mathrm{ha}^{-1}\right)\end{array}$} & \multirow[b]{3}{*}{ Timing $^{\mathrm{y}}$} & \multicolumn{3}{|c|}{ Rebel II } & \multicolumn{5}{|c|}{ Reliant } \\
\hline & & & \multicolumn{2}{|c|}{1997} & 1998 & \multicolumn{2}{|c|}{1997} & \multicolumn{3}{|c|}{1998} \\
\hline & & & 19 Nov. & 5 Dec. & 14 Jan. & 19 Nov. & 5 Dec. & 14 Jan. & 6 Feb. & 6 Mar. \\
\hline \multirow[t]{6}{*}{ Ethofumesate } & 1.12 & Oct. & $9.4 \mathrm{a}^{\mathrm{w}}$ & $9.5 \mathrm{a}$ & $7.8 \mathrm{a}-\mathrm{d}$ & $8.9 \mathrm{~b}-\mathrm{e}$ & $9.1 \mathrm{a}-\mathrm{c}$ & $8.0 \mathrm{a}-\mathrm{d}$ & $7.6 \mathrm{ab}$ & $7.3 \mathrm{ab}$ \\
\hline & 1.12 & Nov. & $8.6 \mathrm{c}$ & $8.8 \mathrm{~cd}$ & $7.8 \mathrm{a}-\mathrm{d}$ & $9.3 \mathrm{ab}$ & $8.5 \mathrm{c}-\mathrm{e}$ & $8.1 \mathrm{ab}$ & $7.8 \mathrm{a}$ & $7.3 \mathrm{ab}$ \\
\hline & 2.24 & Oct. & $9.3 \mathrm{a}$ & $9.1 \mathrm{a}-\mathrm{d}$ & $7.7 \mathrm{c}-\mathrm{e}$ & $6.9 \mathrm{~g}$ & $7.0 \mathrm{f}$ & $7.1 \mathrm{f}$ & $6.3 \mathrm{~d}$ & $6.4 \mathrm{~d}$ \\
\hline & 2.24 & Nov & $7.8 \mathrm{~d}$ & $7.8 \mathrm{e}$ & $7.9 \mathrm{ab}$ & $8.2 \mathrm{f}$ & $6.8 \mathrm{f}$ & $7.4 \mathrm{ef}$ & $6.9 \mathrm{~cd}$ & $7.0 \mathrm{a}-\mathrm{d}$ \\
\hline & $0.56+0.56$ & Oct. + Nov. ${ }^{x}$ & $9.0 \mathrm{a}-\mathrm{c}$ & $9.1 \mathrm{a}-\mathrm{d}$ & $7.9 \mathrm{ab}$ & $9.1 \mathrm{a}-\mathrm{c}$ & $9.0 \mathrm{a}-\mathrm{c}$ & $8.0 \mathrm{a}-\mathrm{d}$ & $7.6 \mathrm{a}-\mathrm{c}$ & $7.1 \mathrm{a}-\mathrm{c}$ \\
\hline & $0.84+0.84$ & Oct. + Nov. ${ }^{x}$ & $8.7 \mathrm{bc}$ & $9.0 \mathrm{~b}-\mathrm{d}$ & $7.9 \mathrm{a}-\mathrm{c}$ & $8.5 \mathrm{~d}-\mathrm{f}$ & $7.9 \mathrm{e}$ & $7.6 \mathrm{e}$ & $7.1 \mathrm{bc}$ & $6.9 \mathrm{a}-\mathrm{d}$ \\
\hline Ethofumesate + prodiamine & $0.84+0.36$ & Oct. & $9.1 \mathrm{ab}$ & $9.1 \mathrm{a}-\mathrm{d}$ & $7.7 \mathrm{~b}-\mathrm{e}$ & $9.2 \mathrm{a}-\mathrm{c}$ & $9.2 \mathrm{ab}$ & $8.0 \mathrm{a}-\mathrm{c}$ & $7.7 \mathrm{ab}$ & $7.1 \mathrm{a}-\mathrm{c}$ \\
\hline Ethofumesate + prodiamine & $1.12+0.73$ & Oct. & $9.1 \mathrm{ab}$ & $9.5 \mathrm{a}$ & $7.5 \mathrm{e}$ & 8.4 ef & $8.6 \mathrm{~b}-\mathrm{d}$ & $7.6 \mathrm{de}$ & $7.0 \mathrm{bc}$ & $6.5 \mathrm{~cd}$ \\
\hline Ethofumesate + prodiamine & $0.84+0.36$ & Nov. & $8.8 \mathrm{bc}$ & $8.8 \mathrm{~d}$ & $8.0 \mathrm{a}$ & $8.7 \mathrm{c}-\mathrm{f}$ & $8.6 \mathrm{~b}-\mathrm{d}$ & $7.6 \mathrm{de}$ & $7.0 \mathrm{bc}$ & $6.5 \mathrm{~cd}$ \\
\hline Ethofumesate + prodiamine & $1.12+0.73$ & Nov & $8.7 \mathrm{bc}$ & $8.8 \mathrm{~cd}$ & $7.9 \mathrm{ab}$ & $9.0 \mathrm{a}-\mathrm{d}$ & $8.0 \mathrm{de}$ & $7.7 \mathrm{c}-\mathrm{e}$ & $7.0 \mathrm{~b}-\mathrm{d}$ & $6.5 \mathrm{~cd}$ \\
\hline Prodiamine & 0.73 & Oct. & $9.1 \mathrm{ab}$ & $9.3 \mathrm{a}-\mathrm{c}$ & $7.6 \mathrm{de}$ & $9.5 \mathrm{a}$ & $9.0 \mathrm{a}-\mathrm{c}$ & $7.8 \mathrm{~b}-\mathrm{e}$ & $7.2 \mathrm{a}-\mathrm{c}$ & $6.9 \mathrm{a}-\mathrm{d}$ \\
\hline Prodiamine & 0.73 & Nov. & $9.3 \mathrm{a}$ & $9.5 \mathrm{a}$ & $7.7 \mathrm{c}-\mathrm{e}$ & $9.3 \mathrm{ab}$ & $9.1 \mathrm{a}-\mathrm{c}$ & $8.0 \mathrm{a}-\mathrm{d}$ & $7.3 \mathrm{a}-\mathrm{c}$ & $6.3 \mathrm{~b}-\mathrm{d}$ \\
\hline Not treated & --- & --- & $9.4 \mathrm{a}$ & $9.3 \mathrm{ab}$ & $7.8 \mathrm{a}-\mathrm{d}$ & $9.5 \mathrm{a}$ & $9.5 \mathrm{a}$ & $8.3 \mathrm{a}$ & $7.8 \mathrm{a}$ & $7.3 \mathrm{a}$ \\
\hline
\end{tabular}

${ }^{2}$ Turf quality was assessed visually using a 0 to 10 scale where $0=$ entire plot area brown or dead, and $10=$ optimum color and density.

y Herbicides were applied on either 8 Oct. or 4 Nov. 1997.

${ }^{x}$ Herbicides were applied to the same plots on 8 Oct. and 4 Nov. 1997.

"Mean separation within columns by LSD, $P \leq 0.05$.

Table 4. 'Jamestown II' Chewings fescue and 'MX 86' blue sheep fescue quality ${ }^{\mathrm{z}}$ as influenced by ethofumesate and prodiamine application, 1997 and 1998.

\begin{tabular}{|c|c|c|c|c|c|c|c|c|c|c|c|c|}
\hline \multirow[b]{3}{*}{ Treatment } & \multirow{3}{*}{$\begin{array}{c}\text { Rate } \\
\left(\mathrm{kg} \cdot \mathrm{ha}^{-1}\right)\end{array}$} & \multirow[b]{3}{*}{ Timing $^{\mathrm{y}}$} & \multicolumn{5}{|c|}{ Jamestown II } & \multicolumn{5}{|c|}{ MX 86} \\
\hline & & & \multirow{2}{*}{$\frac{1997}{19 \text { Nov. }}$} & \multicolumn{4}{|c|}{1998} & \multicolumn{2}{|c|}{1997} & \multicolumn{3}{|c|}{1998} \\
\hline & & & & 14 Jan. & 6 Feb. & 2 Apr. & 4 May & 19 Nov. & 5 Dec. & 6 Feb. & 6 Mar. & 4 May \\
\hline \multirow[t]{6}{*}{ Ethofumesate } & 1.12 & Oct. & $8.8{b c^{w}}^{w}$ & $8.1 \mathrm{a}-\mathrm{d}$ & $7.1 \mathrm{c}$ & $7.8 \mathrm{c}$ & $7.7 \mathrm{~d}$ & $8.8 b^{x}$ & $8.5 \mathrm{~cd}$ & $6.3 \mathrm{c}-\mathrm{e}$ & $5.9 \mathrm{c}$ & $6.8 \mathrm{c}$ \\
\hline & 1.12 & Nov. & $9.0 \mathrm{a}-\mathrm{c}$ & $7.7 \mathrm{de}$ & $7.9 \mathrm{a}$ & $8.7 \mathrm{ab}$ & $8.3 \mathrm{a}-\mathrm{c}$ & $8.9 \mathrm{a}-\mathrm{c}$ & $7.8 \mathrm{de}$ & $7.0 \mathrm{a}$ & $6.9 \mathrm{ab}$ & $7.8 \mathrm{a}$ \\
\hline & 2.24 & Oct. & $6.9 \mathrm{e}$ & $7.5 \mathrm{e}$ & $6.2 \mathrm{~d}$ & $6.7 \mathrm{~d}$ & $6.7 \mathrm{e}$ & $6.1 \mathrm{e}$ & $6.2 \mathrm{f}$ & $5.3 \mathrm{f}$ & $5.3 \mathrm{~d}$ & $5.9 \mathrm{~d}$ \\
\hline & 2.24 & Nov. & $7.5 \mathrm{f}$ & $6.9 \mathrm{f}$ & $7.2 \mathrm{c}$ & $8.1 \mathrm{bc}$ & $8.2 \mathrm{a}-\mathrm{d}$ & $7.8 \mathrm{~d}$ & $6.1 \mathrm{f}$ & $6.0 \mathrm{e}$ & $6.0 \mathrm{c}$ & $7.0 \mathrm{bc}$ \\
\hline & $0.56+0.56$ & Oct. + Nov. ${ }^{x}$ & $9.0 \mathrm{a}-\mathrm{c}$ & $8.2 \mathrm{a}-\mathrm{c}$ & $7.8 \mathrm{ab}$ & $8.6 \mathrm{ab}$ & $8.5 \mathrm{a}$ & $9.0 \mathrm{a}-\mathrm{c}$ & $8.3 \mathrm{~d}$ & $6.5 \mathrm{~b}-\mathrm{d}$ & $6.5 \mathrm{ab}$ & $7.5 \mathrm{ab}$ \\
\hline & $0.84+0.84$ & Oct. + Nov. ${ }^{\mathrm{x}}$ & $8.5 \mathrm{c}$ & $7.7 \mathrm{de}$ & $7.5 \mathrm{a}-\mathrm{c}$ & $8.2 \mathrm{bc}$ & $7.8 \mathrm{~cd}$ & $8.6 \mathrm{c}$ & $7.2 \mathrm{e}$ & $6.0 \mathrm{~d}$ & $6.4 \mathrm{bc}$ & $7.6 \mathrm{ab}$ \\
\hline $\begin{array}{c}\text { Ethofumesate }+ \\
\text { prodiamine }\end{array}$ & $0.84+0.36$ & Oct. & $9.3 \mathrm{ab}$ & $8.3 \mathrm{ab}$ & $7.6 \mathrm{a}-\mathrm{c}$ & $8.1 \mathrm{bc}$ & $8.0 \mathrm{a}-\mathrm{d}$ & $9.2 \mathrm{a}-\mathrm{c}$ & $8.6 \mathrm{~b}-\mathrm{d}$ & $6.8 \mathrm{a}-\mathrm{c}$ & $6.5 \mathrm{ab}$ & $7.5 \mathrm{a}$ \\
\hline Ethofumesate + & 3 & Oct & $7 \mathrm{hat}$ & 8 & & 8 & 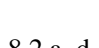 & $8 \mathrm{r}+\mathrm{k}$ & $51+2>$ & $65 \mathrm{~h}$ & & \\
\hline Ethofumesate + & $12+0.13$ & Uct. & $.7 \mathrm{DC}$ & $1.8 \mathrm{c}-\mathrm{e}$ & $1.1 \mathrm{c}$ & $1.8 \mathrm{c}$ & $8.2 \mathrm{a}-\mathrm{d}$ & $8.8 \mathrm{bc}$ & $8.5 \mathrm{~b}-\mathrm{d}$ & $6.5 \mathrm{~b}-\mathrm{e}$ & $6.6 \mathrm{ab}$ & $.8 \mathrm{a}$ \\
\hline prodiamine & $0.84+0.36$ & Nov. & $8.8 \mathrm{bc}$ & $7.9 \mathrm{~b}-\mathrm{d}$ & $7.5 \mathrm{a}-\mathrm{c}$ & $8.5 \mathrm{ab}$ & $8.5 \mathrm{ab}$ & $9.3 \mathrm{ab}$ & $8.4 \mathrm{~d}$ & $6.9 \mathrm{ab}$ & $7.0 \mathrm{a}$ & $7.7 \mathrm{at}$ \\
\hline $\begin{array}{c}\text { Ethofumesate }+ \\
\text { prodiamine }\end{array}$ & $1.12+0.73$ & Nov. & $8.8 \mathrm{bc}$ & $7.6 \mathrm{de}$ & $7.3 \mathrm{c}$ & $8.4 \mathrm{ab}$ & $8.0 \mathrm{~b}-\mathrm{d}$ & $9.2 \mathrm{a}-\mathrm{c}$ & $8.1 \mathrm{~d}$ & $6.9 \mathrm{ab}$ & $6.8 \mathrm{ab}$ & $7.5 \mathrm{a}$ \\
\hline Prodiamine & 0.73 & Oct. & $9.3 \mathrm{ab}$ & $8.0 \mathrm{a}-\mathrm{d}$ & $7.5 \mathrm{a}-\mathrm{c}$ & $8.3 \mathrm{a}-\mathrm{c}$ & $8.3 \mathrm{ab}$ & $9.4 \mathrm{ab}$ & $9.3 \mathrm{ab}$ & $6.8 \mathrm{a}-\mathrm{c}$ & $6.6 \mathrm{ab}$ & $7.6 \mathrm{ab}$ \\
\hline Prodiamine & 0.73 & Nov. & $9.3 \mathrm{ab}$ & $8.0 \mathrm{~b}-\mathrm{d}$ & $7.4 \mathrm{bc}$ & $8.2 \mathrm{bc}$ & $8.0 \mathrm{a}-\mathrm{d}$ & $9.5 \mathrm{a}$ & $9.5 \mathrm{a}$ & $7.0 \mathrm{ab}$ & $6.9 \mathrm{ab}$ & $7.6 \mathrm{ab}$ \\
\hline Not treated & --- & --- & $9.4 \mathrm{a}$ & $8.4 \mathrm{a}$ & $7.9 \mathrm{a}$ & $8.9 \mathrm{a}$ & $8.0 \mathrm{~b}-\mathrm{d}$ & $9.5 \mathrm{a}$ & $9.2 \mathrm{a}-\mathrm{c}$ & $7.1 \mathrm{a}$ & $6.9 \mathrm{ab}$ & $7.6 \mathrm{ab}$ \\
\hline
\end{tabular}

${ }^{2}$ Turf quality was assessed visually using a 0 to 10 scale where $0=$ entire plot area brown or dead, and $10=$ optimum color and density.

y Herbicides were applied on either 8 Oct. or 4 Nov. 1997.

${ }^{x}$ Herbicides were applied to the same plots on 8 Oct. and 4 Nov. 1997.

${ }^{w}$ Mean separation within columns by LSD, $P \leq 0.05$ 
the other species; however, objectionable discoloration occurred with single applications $\geq 1.12 \mathrm{~kg} \cdot \mathrm{ha}^{-1}$ or sequential rates of $\geq 0.84+$ $0.84 \mathrm{~kg} \cdot \mathrm{ha}^{-1}$ (Table 1). The $2.24+2.24 \mathrm{~kg} \cdot \mathrm{ha}^{-1}$ treatment caused commercially unacceptable discoloration. The ETHO label allows for up to three applications at $2.24 \mathrm{~kg} \cdot \mathrm{ha}^{-1}$, however, this obviously would have been too injurious to 'Rebel II'. 'Reliant' appeared to tolerate ETHO better than either 'Jamestown II' or 'MX 86'. In Study I, 'Reliant' showed little injury following a single application of ETHO at 0.56 to $2.24 \mathrm{~kg} \cdot \mathrm{ha}^{-1}$ (Table 1 ). The sequential treatments, however, caused unacceptable quality and the $2.24+2.24 \mathrm{~kg} \cdot \mathrm{ha}^{-1}$ rate significantly reduced 'Reliant' cover (3\% bareground). In Study II, only $2.24 \mathrm{~kg} \cdot \mathrm{ha}^{-1}$ caused unacceptable 'Reliant' quality on two or more rating dates (Table 4); however, plots had recovered fully by 4 May (data not shown).

'Jamestown II' was very sensitive to ETHO, and only $0.56 \mathrm{~kg} \cdot \mathrm{ha}^{-1}$ applied once did not elicit injury in Study I (Table 2). While the sequential $0.56+0.56 \mathrm{~kg} \cdot \mathrm{ha}^{-1}$ reduced quality on a few dates in Study I, sequential rates $\geq 0.84+0.84 \mathrm{~kg} \cdot \mathrm{ha}^{-1}$ reduced quality to unacceptable levels (ratings $\leq 5.3$ ). Following treatment with $1.12+1.12$ and $2.24+2.24 \mathrm{~kg} \cdot \mathrm{ha}^{-1}$ 'Jamestown II' did not fully recover, and plots had $8 \%$ and $70 \%$ bareground, respectively. In Study II, only $2.24 \mathrm{~kg} \cdot \mathrm{ha}^{-1}$ caused unacceptable quality (Table 4). Single applications of 1.12 and $2.24 \mathrm{~kg} \cdot \mathrm{ha}^{-1}$ in October generally caused greater injury than did the same treatments applied in November. Only plots treated with $2.24 \mathrm{~kg} \cdot \mathrm{ha}^{-1}$ in October exhibited quality inferior to that of the control on the final rating date (4 May).

The cultivar MX 86 was tolerant of all single ETHO treatments in Study I, but the sequential treatments caused unacceptable injury. In Study II, 1.12 and $2.24 \mathrm{~kg} \cdot \mathrm{ha}^{-1}$ applied once reduced quality to unacceptable levels on selected dates; however, both rates applied in October resulted in unacceptable quality and loss of turf cover (3\% to $4 \%$ bareground) on the final rating date (4 May). In contrast, plots treated in November had recovered by 4 May. The nature of the increased sensitivity of 'MX 86' and 'Jamestown II' to treatment in October is unknown; however, warmer temperatures in October probably allowed for more foliar uptake of the herbicide.

In Maryland, ETHO is most effective in controlling annual bluegrass in perennial ryegrass when applied at $2.24 \mathrm{~kg} \cdot \mathrm{ha}^{-1}$ or sequentially at rates $\geq 1.12+1.12 \mathrm{~kg} \cdot \mathrm{ha}^{-1}$ (Dernoeden and Turner, 1988). While the high, single-application rate $\left(2.24 \mathrm{~kg} \cdot \mathrm{ha}^{-1}\right)$ caused injury, all fescues recovered by spring in Study I, but only plots treated in November had recovered by spring in Study II. In this study, all fescues recovered from the $0.84+0.84$ $\mathrm{kg} \cdot \mathrm{ha}^{-1}$ sequential (October and November) treatment, which would provide some annual bluegrass control, but the level of control probably would be inferior to more frequent applications or the use of higher rates (Branham, 1991; Dernoeden and Turner, 1988; Taylorson and Spak, 1994). Nevertheless, this study indicates that ETHO will probably cause objectionable injury to all fescue species evaluated. These data indicate that ETHO should be used with caution on fine-leaf fescues, and that sequential applications should be avoided. Unless further studies indicate otherwise, ETHO rates $>1.12 \mathrm{~kg} \cdot \mathrm{ha}^{-1}$ probably should not be applied to 'Jamestown II', 'MX 86', or 'Reliant'. Only 'Rebel II' tolerated $1.12+1.12$ $\mathrm{kg} \cdot \mathrm{ha}^{-1}$, but $2.24+2.24 \mathrm{~kg} \cdot \mathrm{ha}^{-1}$ was too injurious to tall fescue. Ethofumesate appears to be safer to apply in November than in October, but corroborative studies need to be performed to verify these observations.
Prodiamine applied preemergence in early to mid-September at $\geq 0.36 \mathrm{~kg} \cdot \mathrm{ha}^{-1}$ provided excellent control of the annual biotype of $\mathrm{Poa}$ annua in Maryland (Dernoeden, 1998). All fescues in this study tolerated prodiamine at $0.73 \mathrm{~kg} \cdot \mathrm{ha}^{-1}$. Therefore, a preemergence application of prodiamine would be the safest and possibly most efficacious method of annual bluegrass control in the fescues. Curiously, however, data also indicated that the fescues could be treated relatively safely with ETHO $\left(0.84\right.$ or $\left.1.12 \mathrm{~kg} \cdot \mathrm{ha}^{-1}\right)$ tank-mixed with prodiamine. 'Reliant', however, was discolored to unacceptable levels by ETHO + prodiamine in Study II on one rating date (6 Mar.). Future research should assess the potential efficacy of ETHO tank-mixed with prodiamine to determine if early postemergence and subsequent preemergence control of annual bluegrass can be achieved safely in the fescues.

\section{Literature Cited}

Branham, B. 1991. Dealing with Poa апnиа. Golf Course Mgt. 59(9):46, 48, 52, 54, 58, 60.

Dernoeden, P.H. 1998a. Use of prodiamine as a preemergence herbicide to control annual bluegrass in Kentucky bluegrass. HortScience 33:845-846.

Dernoeden, P.H. 1998b. Fine fescues on golf courses. Golf Course Mgt. 66(4):56-60.

Dernoeden, P.H., M.A. Fidanza, and J.M. Krouse. 1998. Low maintenance performance of five Festuca species in monostands and mixtures. Crop Sci. 38:434-439.

Dernoeden, P.H. and T.T. Turner. 1988. Annual bluegrass control and tolerance of Kentucky bluegrass and perennial ryegrass to ethofumesate. HortScience 23:565-567.

Taylorson, R.B. and D.R. Spak. 1994. Control of annual bluegrass with ethofumesate-Field studies and effects of biotype and growth stage. Proc. Northeastern Weed Sci. Soc. 48:123. (Abstr.) 